(1)

CrossMark

\title{
Oral steroids in asthma: a double-edged sword
}

\author{
Mina Gaga (1) and Eleftherios Zervas (1)
}

Affiliation: 7th Respiratory Medicine Dept and Asthma Center, Athens Chest Hospital Sotiria, Athens, Greece.

Correspondence: Mina Gaga, 7th Respiratory Medicine Dept and Asthma Center, Athens Chest Hospital Sotiria, 152 Mesogion Ave, Athens 11527, Greece. E-mail minagagadyahoo.com

@ERSpublications

There is poor evidence on the benefits of long-term systemic steroid use in asthma while the risks of morbidity and mortality are high. Systemic steroids should be a last resort and should be withdrawn in non-responders. http://bit.ly/343fs3T

Cite this article as: Gaga M, Zervas E. Oral steroids in asthma: a double-edged sword. Eur Respir J 2019; 54: 1902034 [https://doi.org/10.1183/13993003.02034-2019].

Since the introduction of inhaled steroids, the management of asthma has been revolutionised and the lives of millions of patients have improved. Nevertheless, systemic corticosteroids (SCS), usually prescribed as oral treatment, but which can also be prescribed for intravenous and intramuscular administration, are still quite extensively used, both for the management of severe asthma exacerbations and, in many cases, for the long-term management of difficult to treat and severe asthma.

Is the use of systemic steroids justified? And what are the short- and long-term risks associated with their use? Musculoskeletal, gastrointestinal, cardiovascular, endocrine, psychiatric, ocular, dermatological and immunological side-effects are all possible, and there is undisputable evidence linking long-term SCS use with infection, osteoporosis and bone fractures, cataracts, adrenal failure, diabetes, and hypertension, to name some of these side-effects [1]. Increased use of healthcare services has also been reported, and this can be associated to both the severity of the underlying disease as well as SCS side-effects. Moreover, there is evidence linking SCS use with mortality in various diseases: in a prospective study that followed of 5600 rheumatoid arthritis patients for a median of 4.7 years, prednisone was associated with a 2.83 hazard ratio for mortality [2]. In a much smaller study following 52 moderate-severe asthma patients for 20 years, the authors also report increased risk of mortality associated with systemic steroid use in asthma [3].

In this issue of the European Respiratory Journal, LEE et al. [4] examine more than 8000 cases of SCS-dependent asthma and report that chronic SCS use is associated with increased risk of mortality (hazard ratio 2.17). They also report that there is a significant dose-response relationship; that is, the higher the dose of steroids, the higher the risk.

LEE et al. [4] performed a population-based matched cohort study using the Korean National Health Insurance Service database from 2005 to 2015. The importance of the study comes with the enormous database, a total of 466941 patients with asthma, of whom 8334 were SCS-dependent. Considering that severe asthma comprises $2-10 \%$ of all asthma and that $20-60 \%$ of severe asthma patients receive SCS, the numbers appear correct [5-7]. It is of course difficult to retrospectively identify asthma patients and, moreover, severe asthma patients, using pre-existing electronic records. This may be considered a problem in the study but defining asthma for an epidemiological study is always a problem, even in large prospective trials such as the European Community Respiratory Health Survey [8]. Epidemiological definitions are based on symptoms indicative of asthma, medication prescriptions and a doctor diagnosis 
of asthma, and should exclude COPD or other diseases with similar symptoms. The definition of asthma that the authors used in order to identify asthmatic patients in the electronic records included the asthma patients with two or more visits and asthma-related medication under ICD-10 codes J45-J46 and, furthermore, to identify severe SCS-dependent asthma, the definition included those with at least 6 months of SCS use during a period of 12 months at baseline (prior to entry to the study). The authors compared the severe group to a 1:1 matched non-severe subgroup of the cohort. It must be taken into account that the comparator group was not comprised entirely of patients with mild asthma; it included patients with up to 6 months of SCS prescriptions in a year. So, some patients in the comparator group had quite important disease load as well as SCS load too but, still, there was significantly higher risk of mortality in the severe asthma group receiving SCS more than $50 \%$ of the days per year. Moreover, compared with the SCS-independent cohort, more patients in the SCS-dependent cohort had significantly higher rates of comorbidities, which included diabetes mellitus, Cushing's syndrome, adrenal insufficiency, osteoporosis, vertebral or pelvic bone fractures, pneumonia, tuberculosis and congestive heart failure. Information on steroid bursts was not included in the database and therefore not analysed; the SCS-dependent asthma patients were nevertheless classified into high- and low-dose groups based on the median daily doses of SCS used. Low-dose SCS was defined as less than a median of $5.5 \mathrm{mg}$ per day of a prednisolone equivalent dose. Both high- and low-dose SCS groups had higher risk of comorbidity and mortality, and the risk increased with the higher SCS dosing.

Although SCS bursts are used in severe asthma exacerbations, whether the use of SCS is justified long term and whether it actually helps control of the disease is a matter of debate [9]. This is a question that is currently receiving a lot of attention. There are very few studies examining the effect of long-term use of steroids in asthma and identifying which are the patients who may benefit from their use. In the SHARP cohort, a total of $21 \%$ of adults with severe asthma and $20 \%$ of children with severe asthma achieved greater than or equal to $10 \%$ improvement after intramuscular use of triamcinolone [10]. Similarly, in a paediatric severe asthma population including 102 children with severe asthma, only $11 \%$ of these children exhibited complete corticosteroid responsiveness [11]. In a subsequent study from the same group, the authors examined 56 children at baseline and 14 days after intramuscular triamcinolone injection and used the Asthma Control Questionnaire to classify children with severe asthma into corticosteroid response groups [12]. They also examined various other outcomes: asthma-related quality of life, exhaled nitric oxide, blood eosinophils, lung function, and inflammatory cytokine and chemokine mRNA gene expression in peripheral blood mononuclear cells, Only $13 \%$ of the children had complete response to steroids, $72 \%$ had partial response while $15 \%$ showed no response at all [12]. The authors concluded that response to steroids is inhomogeneous and that alternative models are needed to identify and predict the response. Finally, in the BIOAIR longitudinal cohort, the highest sensitivity and specificity to predict more than $12 \%$ increase in forced expiratory volume in $1 \mathrm{~s}$ in severe asthma after oral prednisolone was found in patients with sputum eosinophils $\geqslant 3 \%$ and exhaled nitric oxide fraction $>45 \mathrm{ppb}$ [13]. These are the T2 high phenotype patients [14], the patients who are candidates for anti-IL5 treatment or perhaps anti-IgE treatment. In the current Global Initiative for Asthma guidelines [15], biologicals are the preferred choice for those patients, and this is also the recommendation in the 2014 European Respiratory Society (ERS)/ American Thoracic Society (ATS) and the recently published ERS/ATS guidelines on the management of severe asthma $[16,17]$, where steroid sparing strategies are very important and alternative strategies that avoid or reduce steroid use are recommended.

It seems then that, although the use of oral/systemic corticosteroids is prevalent in severe asthma management, the risks of acute and chronic complications, including death, are important and increase with higher/cumulative SCS dosage. According to a recently published systematic literature review, long-term and repeated short-term oral/systemic corticosteroid use were associated with increased risk of acute and chronic adverse events, even when doses were comparatively low [18]. Greater oral/systemic corticosteroid exposure was also associated with increased costs and healthcare resource utilisation.

SCS responsiveness in severe asthma is heterogeneous. Prior to resorting to SCS, symptom relief and prevention of exacerbations need to be attempted with high-dose inhaled corticosteroid, long-acting beta-agonist and long-acting muscarinic antagonist combinations as well as biologicals, azithromycin and, in some cases, bronchial thermoplasty, as described in the recently published ERS/European Academy of Allergy and Clinical Immunology statement on severe asthma exacerbations [19] or in the algorithmic approach published last year in ERJ Open Research [20]. High-dose and frequent steroid bursts leading to high cumulative doses and long-term systemic steroid management in asthma need really to be a last resort and the response to this treatment needs to be carefully and continuously monitored. Alternative prediction models that include clinical phenotypic features as well as molecular endotypic ones are needed to identify which patients derive the most clinical benefit from SCS step-up therapy, given the potential side-effects and risks associated with steroid use. When resorting to systemic steroids is considered 
necessary, the patient's response needs to be monitored meticulously. And, considering the side-effects and risks, discontinuation of SCS needs to be attempted in patients with no or minimal response.

Conflict of interest: M. Gaga reports grants and personal fees from AZ, grants from BI, Elpen, Novartis and Menarini, personal fees from BMS, MSD, Chiesi and Pharmaten, outside the submitted work. E. Zervas reports personal fees consultancy and lectures from Astra, Bristol-Myers Squibb, Chiesi, GSK, Elpen, Merck, MSD, Novartis, Menarini and Pfizer, non-financial support for travel, accommodation and meeting expenses from Astra, Bristol-Myers Squibb, Galenica, Chiesi, Elpen, Novartis, Menarini and Roche, outside the submitted work.

\section{References}

1 Volmer T, Effenberger T, Trautner C, et al. Consequences of long-term oral corticosteroid therapy and its side-effects in severe asthma in adults: a focused review of the impact data in the literature. Eur Respir J 2018; 52: 1800703.

2 Chester Wasko M, Dasgupta A, Ilse Sears G, et al. Prednisone use and risk of mortality in patients with rheumatoid arthritis: moderation by use of disease-modifying antirheumatic drugs. Arthritis Care Res (Hoboken) 2016; 68: 706-710.

3 Bourdin A, Molinari N, Vachier I, et al. Mortality: a neglected outcome in OCS-treated severe asthma. Eur Respir J 2017; 50: 1701486.

4 Lee H, Ryu J, Nam E, et al. Increased mortality in patients with corticosteroid-dependent asthma: a nationwide population-based study. Eur Respir J 2019; 54: 1900804.

5 Schleich F, Brusselle G, Louis R, et al. Heterogeneity of phenotypes in severe asthmatics. The Belgian Severe Asthma Registry (BSAR). Respir Med 2014; 108: 1723-1732.

6 Heaney LG, Brightling CE, Menzies-Gow A, et al. Refractory asthma in the UK: cross-sectional findings from a UK multicentre registry. Thorax 2010; 65: 787-794.

7 Kim M-H, Kim S-H, Park S-Y, et al. Characteristics of adult severe refractory asthma in Korea analyzed from the severe asthma registry. Allergy Asthma Immunol Res 2019; 11: 43-54.

8 Burney PG, Luczynska C, Chinn S, et al. The European Community Respiratory Health Survey. Eur Respir J 1994; 7: 954-960.

9 Ganaie MB, Munavvar M, Gordon M, et al. Patient- and parent-initiated oral steroids for asthma exacerbations. Cochrane Database Syst Rev 2016; 12: CD012195.

10 Phipatanakul W, Mauger DT, Sorkness RL, et al. Effects of age and disease severity on systemic corticosteroid responses in asthma. Am J Respir Crit Care Med 2017; 195: 1439-1448.

11 Bossley CJ, Saglani S, Kavanagh C, et al. Corticosteroid responsiveness and clinical characteristics in childhood difficult asthma. Eur Respir J 2009; 34: 1052-1059.

12 Bossley CJ, Fleming L, Ullmann N, et al. Assessment of corticosteroid response in pediatric patients with severe asthma by using a multidomain approach. J Allergy Clin Immunol 2016; 138: 413-420.

13 Kupczyk M, Haque S, Middelveld RJ, et al. Phenotypic predictors of response to oral glucocorticosteroids in severe asthma. Respir Med 2013; 107: 1521-1530.

14 Woodruff PG, Modrek B, Choy DF, et al. Thelper type 2-driven inflammation defines major subphenotypes of asthma. Am J Respir Crit Care Med 2009; 180: 388-395.

15 Global Initiative for Asthma (GINA). Global Strategy for Asthma Management and Prevention. 2019. Available from: http://ginasthma.org/

16 Chung KF, Wenzel SE, Brozek JL, et al. International ERS/ATS guidelines on definition, evaluation and treatment of severe asthma. Eur Respir J 2014; 43: 343-373.

17 Holguin F, Cardet JC, Chung KF, et al. Management of severe asthma: a European Respiratory Society/American Thoracic Society guideline. Eur Respir J 2019; in press [https://doi.org/10.1183/13993003.00588-2019].

18 Bleecker ER, Menzies-Gow AN, Price DB, et al. Systematic literature review of systemic corticosteroid use for asthma management. Am J Respir Crit Care Med 2019; in press [https://doi.org/10.1164/rccm.201904-0903SO].

19 Bourdin A, Bjermer L, Brightling C, et al. ERS/EAACI statement on severe exacerbations in asthma in adults: facts, priorities and key research questions. Eur Respir J 2019; 54: 1900900.

20 Zervas E, Samitas K, Papaioannou AI, et al. An algorithmic approach for the treatment of severe uncontrolled asthma. ERJ Open Res 2018; 4: 00125-2017. 